\title{
Convective Heat Loss Analysis of a Cavity Receiver for a Solar Concentrator
}

\author{
O. López ${ }^{1}$, A. Arenas ${ }^{2}$, and A. Baños ${ }^{1}$ \\ ${ }^{1}$ Dpt. de Electromagnetismo y Electrónica, \\ University of Murcia, \\ Campus de Espinardo - 30071 Murcia (Spain). \\ Contact e-mail: arenas@um.es \\ ${ }^{2}$ Dpt. de Informática y Sistemas, \\ University of Murcia, \\ Campus de Espinardo - 30071 Murcia (Spain). \\ Contact e-mail: A. Baños abanos@um.es, O. López ole52635@um.es
}

\begin{abstract}
The convective heat loss of a modified cavity receiver for a low-cost concentrating solar power plant is studied. Convection is modelled by using the fundamental mass, momentum and energy conservation principles together with the $k$-w SST for turbulence modelling. It is also compared to a flat receiver design. The temperature at the cavity walls is fixed to a non-uniform distribution computed from the solar irradiation field at the walls obtained from a previous work. The convective heat loss rate is computed at different conditions of inclination angle and wind speed of the receivers. As it was expected the cavity improves to the flat receiver; however it is shown that the cavity losses are still considerable mainly due to the wind entering the cavity.
\end{abstract}

\section{Key words}

Forced Convection, Natural Convection, Heat Loss, Solar Collector, Cavity Receiver, Azimuthal Position.

\section{Introduction}

The availability of technologies for the efficient exploitation of renewable energies has become a demand in the energy market. Conventional energy sources, such as fossil and nuclear energy, are still necessary. Nevertheless a reduction of its use has been devised by using renewable sources. The effort in the development of renewable energy technologies is being focused in designing cost-effective facilities to turn renewable energy sources into a feasible energy $[1,2]$.

The technology studied in this work is the Parabolic Dish Concentrator (PDC) technology. Concretely a low power system already deployed for steam generation. A sketch of the PDC system under study can be seen in Fig. 1.

The receiver is where the transformation of radiant to thermal energy occurs. Thermal oil is used as working fluid to transport thermal energy to the boiler, as it is done in [3]. This avoids the phase change inside the receiver that is difficult to control and stabilize [4]. Moreover the transport of superheated steam through large distances can be subjected to a considerable temperature drop.

Convective heat loss is a considerable energy loss in the receivers as it is shown in [5]. Cavity receivers are usually developed with the aim to reduce convective and radiation thermal losses.

This paper extends the work in [6]. The purpose is to analyse the convective heat loss from two different receiver types under different operating conditions of wind and inclination. The receivers considered are a cavity receiver and a flat receiver. The cavity receiver is a cylindrical cavity modified with a curved bottom and dimensioned for its use in low to medium power plants, see Fig. 1 and 2.

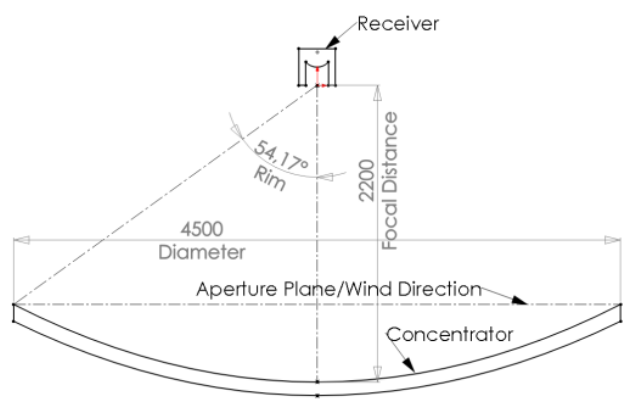

Fig. 1. Sketch of the parabolic dish concentrator under study. The length unit is the millimetre. The focal distance is $2.2 \mathrm{~m}$ and the concentrator radius is $2.25 \mathrm{~m}$, giving an intercepting area of $15.9 \mathrm{~m}^{2}$ 


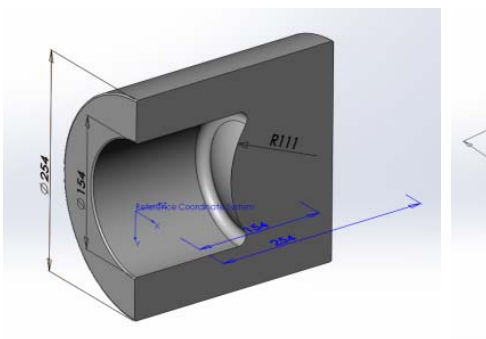

a) Cavity receiver

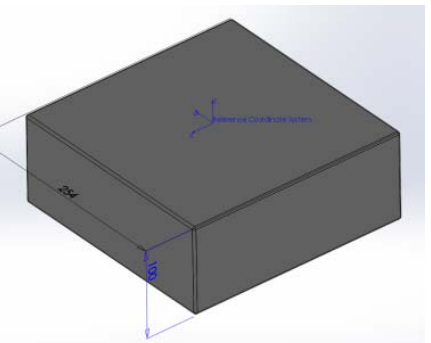

b) Flat receiver

Fig. 2. Sketch of the two solar receivers studied. The length unit is the millimetre

\section{Process Modelling}

The Navier-Stokes equations are employed to model the convective heat transfer phenomena. These include the mass, momentum and energy conservation principles for a continuum medium, together with some constitutive relations and parameters characteristic of a particular medium. The following variables are used to describe the flow: velocity $v$, density $\rho$, pressure $p$, temperature $T$, and enthalpy $h$.

For forced convection or large temperature differences, further modelling is employed, as discussed in the following section.

\section{A. Turbulence modelling for the receivers}

For the receivers the flow becomes turbulent even for moderate wind speeds $(\sim 15 \mathrm{~km} / \mathrm{h})$. In these cases a considerable variation of flow quantities in space and time happens, and obtaining a solution to the flow equations would require a considerable amount of computational resources that is not currently affordable.

Turbulent motion can be modelled by means of some additional transported properties accounting for turbulent effects in the mean flow [7]. Since the mean flow is expected to be smoother, it can help to reduce the computational costs while keeping a reasonable accuracy on the quantities of interest. Concretely, the ReynoldsAverage Navier-Stokes (RANS) approach is chosen here due to being the lowest computationally demanding one.

In a RANS model, at a position $r$ and in an instant $\mathrm{t}$ the value of a flow variable $\phi$ is expressed as $\phi(r, t)=\bar{\phi}(r)+\phi^{\prime}(r, t)$, where $\bar{\phi}$ is the time-mean value and $\phi^{\prime}$ is the instantaneous deviation. For the cases considered here the turbulent motion is stationary; thus the time-average of the flow field, $\bar{\phi}(r)$, exits.

In particular, the $k$-w SST (Shear Stress Transport) turbulence model is employed $[8,9]$. The main reasons are the following. Firstly this model can be integrated down to the wall which is appropriate for wall heat transfer calculations as it depends directly on the thermal boundary

layer close to the wall. Secondly it also predicts recirculation and reattachment regions, which affect to the overall heat loss rate by the dragging of the heated air inside the cavity.

This model adds to the fundamental equations two new flow variables: The turbulent kinetic energy $k$, and the specific dissipation $w$, together with two new transport equations governing them. It also adds new flowdependent parameters that ideally would have to be fined-tuned for every application; however the general values are used since they are shown to be suitable for many applications.

\section{B. Air model}

In the range of the operating conditions studied, air can be approximated as an ideal gas. Its dynamic viscosity is model by the Sutherland's law. Thermal expansion coefficient is given by the equation of state. Thermal conductivity and heat capacity are considered constant and uniform. Reference values are computed at the film temperature $T_{f} \equiv 1 / 2\left(T_{\text {wall }}+T_{\text {bulk }}\right)$, and at the normal pressure $p_{0}=1 \mathrm{~atm}$.

\section{Air-Domain Geometry}

The receiver geometry is placed inside a rectangular cuboid representing the air surrounding the receiver at normal operating conditions.

For natural convection the chimney approach is used. Given a characteristic length of the receiver studied, L, the bounding box is constructed with the following dimensions: The upward distance (the distance from the centre to the roof) is $5 \mathrm{~L}$. The downward distance is $3 \mathrm{~L}$. And the side distances are also $3 \mathrm{~L}$.

For forced convection the same dimensions are used.

\section{Boundary Conditions}

The boundary conditions used are briefly discussed in the following.

\section{Velocity}

For natural convection, the atmospheric patches are set to a zero gradient for velocity (Neumann type). This condition represents an external boundary where the air is able to freely enter or exit the domain.

For forced convection, the atmospheric patches are distinguished. At the inlet patch the velocity is fixed (Dirichlet type), otherwise it is set to zero gradient.

Finally, the velocity at the receivers' walls is set to $0 \mathrm{~m} / \mathrm{s}$.

Pressure 
For pressure the atmospheric patches are set to a fixed total pressure equals to the normal pressure. For inlets and walls a zero gradient condition is used.

\section{Temperature}

Temperature on the receiver transmitting walls is fixed at a non-uniform field. For the remaining faces an insulated boundary condition is used (zero gradient).

In the cavity the transmitting walls are the bottom, side and front faces. The total area is $0.117 \mathrm{~m}^{2}$. In the flat receiver the transmitting face is the horizontal face shown in Fig. 2. Its area is $0.065 \mathrm{~m}^{2}$.

The temperature field is computed based on the energy flux that is being irradiated form the concentrator. This flux is obtained from [6]. In the following the assumptions used for constructing the temperature field are detailed for every receiver.

In the cavity receiver, the working fluid flows from the external wall to the bottom uniformly. Assuming a stationary condition the energy flux can be related to the temperature variation along the symmetry axis, $z$, by $\dot{Q}=C d T / d z$, where $C$ is a constant that can be determined by fixing the operating temperatures of the working fluid. Thus given an inlet temperature $T_{i}$, and an outlet temperature, $T_{0}$, the following expression can be used to calculate the temperature distribution:

$$
T(z)=T_{i}+\frac{\left(T_{o}-T_{i}\right)}{\dot{Q}_{\max }} \int_{0}^{z} \dot{Q}\left(z^{\prime}\right) d z^{\prime}
$$

On the other hand for the flat receiver, it is considered that the working fluid flows through a solenoid inside its body. By considering that the solenoid path covers the receiving area from one side to the other, an expression similar to that above can be used over a symmetry axis of the transmitting wall.

\section{E. Heat Transfer Computation}

The heat transfer at the walls is calculated based on the following expression:

$$
\dot{Q}=\oint_{\substack{\text { Trasnmitiv } \\ \text { Faces }}} \alpha_{\text {eff }} \frac{\partial h}{\partial n} d s
$$

Where $\alpha_{\text {eff }}=\alpha+\alpha_{\text {turbulent }}$ is the effective thermal diffusivity. This expression is derived from the Fourier's thermal conductivity law assuming a zero gradient pressure normal to the wall, which is the pressure boundary condition used here for fixed walls.

\section{Methodology}

The altitude angles considered are typical of the altitude of the Sun in a latitude of $37^{\circ} 59^{\prime} 13^{\prime \prime} \mathrm{N}$, corresponding to the location of Murcia, Spain. The following altitude values are chosen: $\{40,70\}^{\circ}$.
On the other hand, the wind direction is considered parallel to the concentrator aperture plane. We have considered that these directions play a major role than the perpendicular ones since the concentrator itself speeds down the wind. Two wind speeds are considered here: $\{15,30\} \mathrm{km} / \mathrm{h}$. They are representative of a light breeze and a moderate breeze respectively.

The operating temperatures considered are shown in Table I. These values are representative of a collector of aperture area $15.9 \mathrm{~m}^{2}$, based in the experimental data shown in [4].

Table I. - Temperature parameters

\begin{tabular}{|c|c|}
\hline Parameter & Value \\
\hline $\mathrm{T}_{\mathrm{o}}$ & $250^{\circ} \mathrm{C}$ \\
\hline $\mathrm{T}_{\mathrm{i}}$ & $150^{\circ} \mathrm{C}$ \\
\hline $\mathrm{T}_{\mathrm{amb}}$ & $27^{\circ} \mathrm{C}$ \\
\hline $\mathrm{T}_{\mathrm{f}}$ & $152^{\circ} \mathrm{C}$ \\
\hline
\end{tabular}

The temperature profiles calculated with these input values are shown in Fig 3.

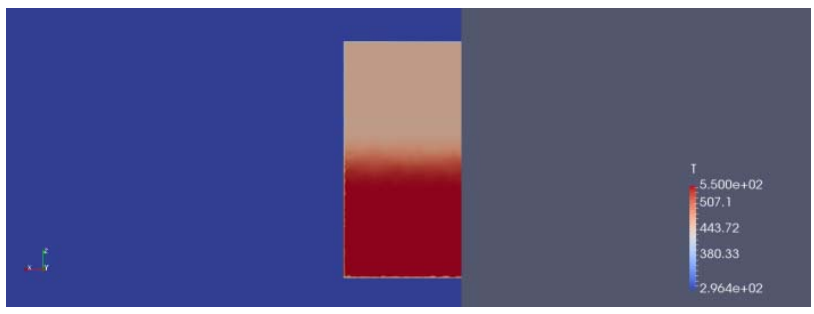

a) Flat receiver

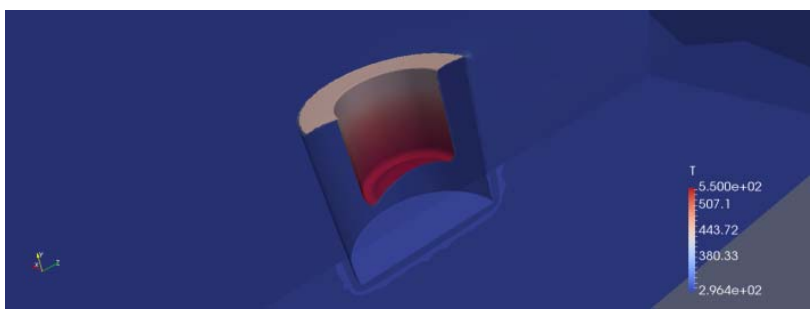

b) Cavity Receiver

Fig. 3. Sketch of the temperature field distribution

These generate the cases summarized in Table II. The Grashof and Reynolds numbers are also shown. For the computation of these values the characteristic lengths used are for the cavity receiver its external diameter, and for the flat receiver its depth.

Table II. - Studied cases

\begin{tabular}{|c|c|c|c|c|c|}
\hline Id & Geometry & $\begin{array}{l}v_{\text {wind }} \\
(\mathrm{km} / \mathrm{h})\end{array}$ & $\begin{array}{l}\text { Altitude } \\
(\mathrm{deg})\end{array}$ & $\begin{array}{c}\text { Reynolds } \\
\text { Number }\end{array}$ & $\begin{array}{c}\text { Grashof } \\
\text { Number }\end{array}$ \\
\hline 1 & Flat & 0 & 40 & - & $9 \cdot 10^{7}$ \\
\hline 2 & Flat & 0 & 70 & - & $9 \cdot 10^{7}$ \\
\hline 3 & Flat & 15 & - & $4 \cdot 10^{4}$ & $9 \cdot 10^{7}$ \\
\hline 4 & Flat & 30 & - & $8 \cdot 10^{4}$ & $9 \cdot 10^{7}$ \\
\hline 5 & Cavity & 0 & 40 & - & $10^{8}$ \\
\hline 6 & Cavity & 0 & 70 & - & $10^{8}$ \\
\hline 7 & Cavity & 15 & - & $2 \cdot 10^{4}$ & $10^{8}$ \\
\hline 8 & Cavity & 30 & - & $7 \cdot 10^{4}$ & $10^{8}$ \\
\hline
\end{tabular}

From Table II, the Grashof numbers fall below the turbulent region $\left(10^{9}\right)$, thus the fundamental equations are 
used. On the other hand, the Reynolds numbers for forced convection are of order $10^{4}-10^{5}$ falling in the transition to turbulence regime. These values are not in the range of high Reynolds numbers for which the turbulence theory is developed; although it is found that these models are also employed at low Reynolds numbers performing well within a reasonable accuracy.

Finally, the atmospheric pressure is set to the normal pressure, $1 \mathrm{~atm}$.

\section{A. Mesh Sensibility Study}

To validate the numerical scheme used, a particular case is simulated for which experimental data is available. The case 1 is chosen for this purpose.

Table III shows the series of meshes studied. In this case the representative magnitude for mesh independency criterion is the rate of change of the total power over the number of elements.

Table III. - Mesh Independency study for case 1. The abbreviations used stand for: FLH for First Layer Height, BL for Boundary Layers, GR for Growth Rate, and ES for Element Size

\begin{tabular}{|c|c|c|c|c|}
\hline $\begin{array}{l}\text { Elements } \\
\left(\cdot 10^{5}\right)\end{array}$ & $\begin{array}{l}\text { FLH } \\
(\mathrm{mm})\end{array}$ & BL & GR & $\begin{array}{l}\text { ES } \\
(\mathrm{mm})\end{array}$ \\
\hline 3.86 & 0.1 & 20 & 1.15 & 7.5 \\
\hline 4.76 & 0.1 & 20 & 1.15 & 5 \\
\hline 11.5 & 0.1 & 15 & 1.15 & 2.5 \\
\hline 26.5 & 0.1 & 10 & 1.15 & 1.5 \\
\hline
\end{tabular}

\begin{tabular}{|c|c|c|}
\hline $\begin{array}{l}\text { Elements } \\
\left(\cdot 10^{5}\right)\end{array}$ & $\begin{array}{l}\text { Power } \\
(\mathrm{W})\end{array}$ & $\begin{array}{c}\text { Rate of change } \\
\left(\mathrm{W} / \text { Element } \cdot 10^{5}\right)\end{array}$ \\
\hline 3.86 & 114 & - \\
\hline 4.76 & 97 & -18.9 \\
\hline 11.5 & 87 & -1.48 \\
\hline 26.5 & 86 & -0.067 \\
\hline
\end{tabular}

It is considered that the third test is independent of the mesh as the rate of change is already below $10^{-5} \mathrm{~W} /$ Element from that number of elements and it is not expected to increase. The element sizes used for this mesh will be used as a guide in the construction of the meshes for the remaining cases.

\section{B. Experimental Validation}

Experimental data is available for inclined walls given as a correlation that can be found in the literature. The correlation used here corresponds to an inclined wall in the laminar regime obtained from [10]. In this case the gravity-inclination correction is used, that is, $g \cos (\varphi)$. In this particular case the inclination angle is $\varphi=40^{\circ}$ and the mean temperature of the heated wall is $\mathrm{T}_{\mathrm{w}}=500 \mathrm{~K}$. The experimental convective heat transfer rate obtained is $82.7 \mathrm{~W}$. Thus, the computed value of the third test from Table III represents a $5 \%$ of relative error from experimental data.

\section{Simulation}

The OpenFOAM ${ }^{\circledR}[11]$ framework has been used for the implementation of the simulations.

\section{A. Natural Convection Simulations}

Natural convection simulations are carried out with the PIMPLE algorithm. Concretely the solver buoyantPimpleFoam is used. For these simulations a time step of $0.1 \mathrm{~s}$ is used.

The final heat transfer value is obtained when it reaches a stationary mean behaviour. It is found to occur at typically above 100 s of simulation time.

\section{B. Forced Convection Simulations}

Forced convection simulations are carried out with the SIMPLE algorithm. The buoyantSimpleFoam solver is used. The convergence criteria are a relative residual of $10^{-3}$ for momentum and continuity equations, and $10^{-6}$ for energy equations.

In these simulations the final heat transfer value is obtained at the stage of convergence. As the iterations advance this value oscillates, and as the stage of convergence is reached they diminish. Despite of the final oscillations only the final value is considered the final result.

\section{Numerical Schemes}

Spatial linear and linear upwind numerical schemes are used, except for velocity divergence where a LUST scheme is used. Time advancement is discretized using the second-order implicit scheme backward.

\section{Results}

The table IV shows the convective heat loss rate for every case. It is also shown the overall heat loss flux over the total receiver transmitting area.

Table IV. - Results

\begin{tabular}{|c|c|c|}
\hline Case Id. & $\begin{array}{l}\text { Heat Loss Rate } \\
(\mathrm{W})\end{array}$ & $\begin{array}{l}\text { Heat Loss Flux } \\
\left(\cdot 10^{3} \mathrm{~W} / \mathrm{m}^{2}\right)\end{array}$ \\
\hline 1 & 87 & 1.39 \\
\hline 2 & 148 & 2.39 \\
\hline 3 & 300 & 4.65 \\
\hline 4 & 472 & 7.31 \\
\hline 5 & 124 & 1.06 \\
\hline 6 & 62 & 0.529 \\
\hline 7 & 352 & 3.01 \\
\hline 8 & 534 & 4.56 \\
\hline
\end{tabular}

Table V shows the relative differences in the overall heat loss flux between the flat and cavity receiver for every case

Table V. - Comparative values

\begin{tabular}{|l|l|l} 
Reference Case & Comparison Case & Relative
\end{tabular}




\begin{tabular}{|c|c|c|}
\hline Id. & Id. & difference \\
\hline 1 & 5 & $27 \%$ \\
\hline 2 & 6 & $75 \%$ \\
\hline 3 & 7 & $35 \%$ \\
\hline 4 & 8 & $38 \%$ \\
\hline
\end{tabular}

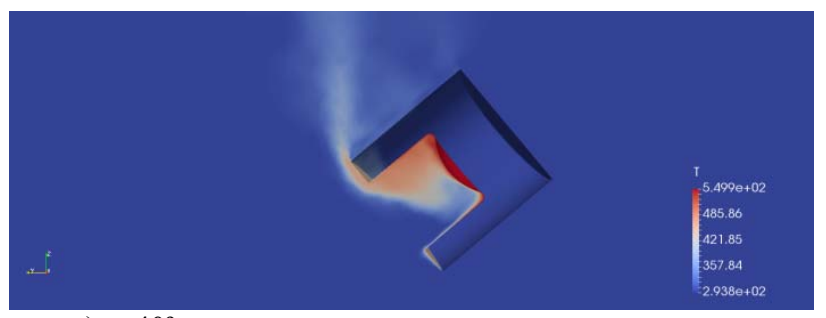

a) $40^{\circ}$

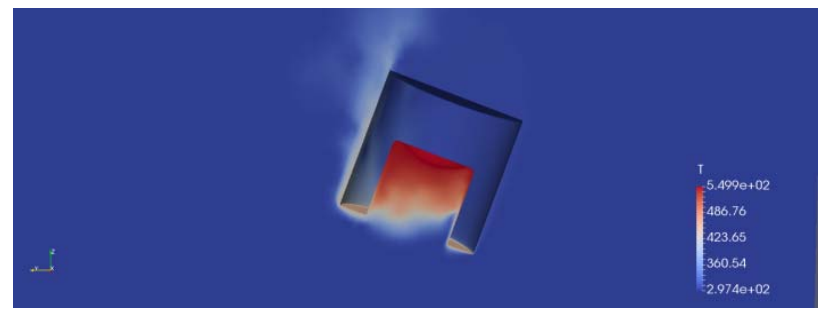

b) $70^{\circ}$

Fig. 4. Temperature field for natural convection in the cavity receiver at 100 s of simulation time for different altitude positions

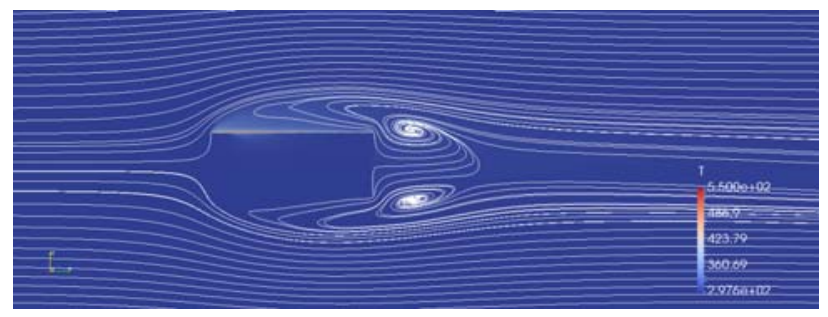

a) Flat receiver

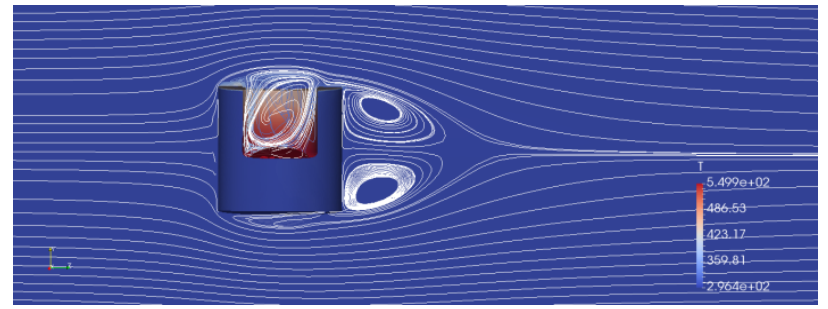

b) Cavity receiver

Fig. 5. Temperature field for forced convection at the state of numerical convergence for a wind speed of $30 \mathrm{~km} / \mathrm{h}$. Flow streamlines are also shown

Natural convection in the cavity receiver is shown in Fig. 4 , where the temperature field at 100 s of simulation time is shown for every inclination. Force convection is shown in Fig. 5 where the mean temperature field and the flow streamlines are shown for a wind speed of $30 \mathrm{~km} / \mathrm{h}$ for every receiver.

\section{Conclusions}

As it can be observed from Table V, the cavity improves the overall heat loss rate, as it was already known. The cavity is more efficient as the angle is increased from $40^{\circ}$ to $70^{\circ}$ which reflects the fact that it can retain more heated air when it approaches to a vertical position, which is the expected inclination of the concentrator in summer. This behaviour is also found in [12]. For forced convection this advantage is reduced since the wind can drag the heated air inside the cavity. This can be observed from Fig. 5b. From Table V it is shown that the improvement is maintained at approximately $35 \%$ for the two wind speeds.

From the results discussed above, the cavity receiver could be improved if a window pane could be placed in the cavity aperture. This could also help to reduce the optical and thermal radiation losses if the glass used were of one-way type.

However, more inclination angles and wind speeds are to be considered for further testing of the trends observed.

\section{Acknowledgement}

This work has been supported by Ministerio de Economia e Innovacion of Spain under project DPI2013-47100-C21-P (including FEDER co-funding).

\section{References}

[1] E. F. Camacho, M. Berenguel, F. R. Rubio, and D. Martínez, Control of Solar Energy Systems, ser. Advances in Industrial Control. Springer London (2012).

[2] I. Dincer and C. Zamfirescu, Advanced Power Generation Systems. Elsevier Science (2014).

[3] L. Valenzuela, E. Zarza, M. Berenguel, and E. F. Camacho, "Direct steam generation in solar boilers," IEEE control systems (2004), pp. 15-29.

[4] J. Dascomb, "Low-cost concentrating solar collector for steam generation," Ph.D. dissertation, Electronic Theses, Teatises and Dissertations (2009), paper 833.

[5] J. A. Harris and T. G. Lenz, "Thermal performance of solar concentrator/cavity receiver systems," Solar Energy (1985), vol. 34, no. 2, pp. $135-142$.

[6] O. López, A. Arenas, and A. Baños, "Radiation performance of a cavity receiver for a parabolic dish solar concentrator system," Renewable Energy \& Power Quality Journal, Vol.1, No.14, May 2016. Paper number 370 .

[7] Wilox, D. C., “Turbulence Modeling for CFD” (Third Edition) D C W Industries (2006).

[8] Menter, F. R., "Zonal Two Equation k- $\omega$ Turbulence Models for Aerodynamic Flows", AIAA Paper 93-2906 (1993).

[9] Menter, F. R., "Two-Equation Eddy-Viscosity Turbulence Models for Engineering Applications", AIAA Journal, vol. 32, no 8. pp. 1598-1605 (1994).

[10] Bejan, A. Convection Heat Transfer, Fourth Edition. Wiley. Duke University, Durham, North Carolina (2013) pp. 203-204.

[11] OpenFOAM ${ }^{\circledR}$ Version 4.0 (2016). OPENFOAM ${ }^{\circledR}$ is a registered trade mark of OpenCFD Limited, producer and distributor of the OpenFOAM software. http://openfoam.com/

[12] N. Sendhil Kumar and K.S. Reddy (2008), "Comparison of receivers for solar dish collector 
system", Energy Conversion and Management, vol. 49, no
4, pp. 812-819. 